\title{
On low frequency disc-brake vibrations
}

\author{
H. Hetzler* and W. Seemann \\ Universität Karlsruhe (TH) - Institut für Technische Mechanik - 76128 Karlsruhe, Germany
}

\begin{abstract}
Vibrations in vehicle disc-brakes cause dissatisfaction of costumers and are therefore subject of intensive research activities. While initially the focus was primarily set on high-frequency squeal noise, it has recently been shifted to more low and medium frequency noise, which is often referred to as groan, chirp oder judder. Within this article experimental results from our test installation are presented. Motivated by the study of these data and a thorough analysis of the functionality of the tested floating caliper disc-brake, a 3-DOF lumped parameter model is developed, which shows very good correspondence to the behavior observed in the experiments. Analysis of this model suggests a direct link between lateral disc-stiffness and the groan frequency, while the circumferential stiffnesses of the pads and the support relate to moan or low frequency squeal.
\end{abstract}

(C) 2005 WILEY-VCH Verlag GmbH \& Co. KGaA, Weinheim

\section{Experimental results}

The noise phenomena exhibited by the test brake reach from low frequency groan to high frequency squeal, depending on the system's state when the vibrations are triggered and the overall operating conditions. In this paper, measurements showing mixed noise are presented. The sound measurements comprise low and medium frequencies, often referred to as groan and muh (low frequency squeal) [1].
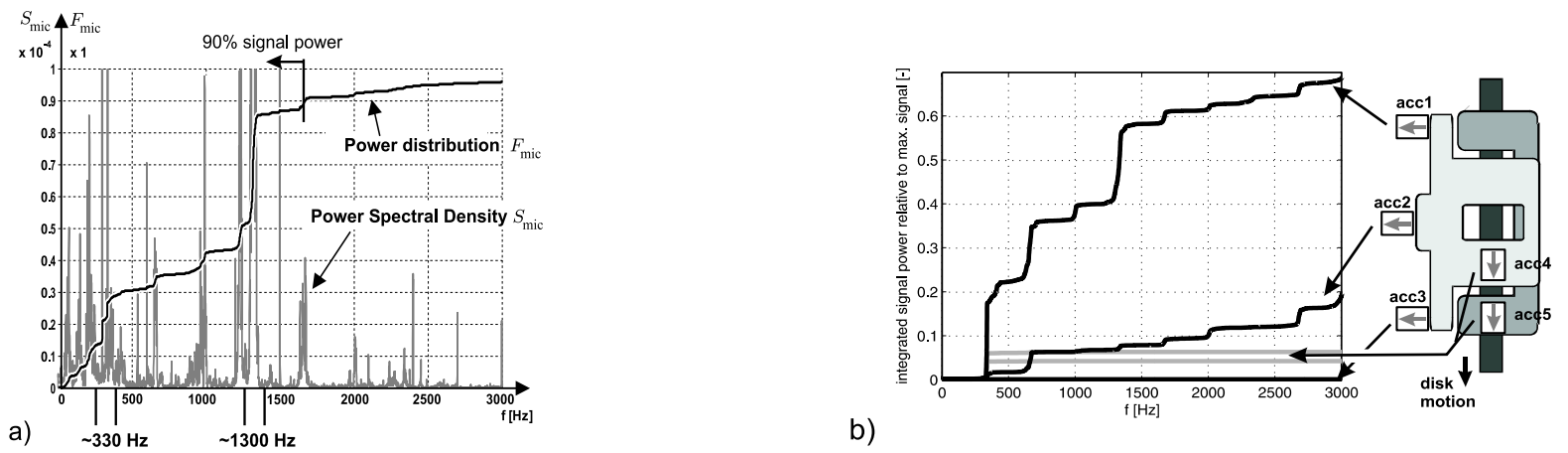

b)

Fig. 1 a) Measurement of microphone data: signal power spectral density (PSD) $S_{m i c}$ and normalized integrated signal power distribution $F_{m i c}(f)=\max \left(S_{m i c}\right)^{-1} \int_{0}^{f} S_{m i c}(f) d f$.

b) Measurement of accelerations: signal power distributions $F_{i}(f)=\int_{0}^{f} S_{i i} d f$, scaled to the maximum of strongest signal. Positions of lateral and circumferential acceleration sensors.

Figure 1a shows the power spectral density (PSD) of the audio measurement. Since the PSD can contain almost singular values, additionally the integrated PSD - which then is finite - is outlined to give a better overview. For a particular channel $i$ and its PSD $S_{i i}(f)$, the integral $F_{i}(f)=\int_{0}^{f} S_{i i} d f$ gives the average power of the signal in the frequency band $[0, f]$ Hertz. In analogy to stochastics, this integral can be seen as an unnormalized distribution of the spectral power. Since steep inclinations of $F_{i}$ denote frequency bands, which introduce much power to the signal, strong energy input to the acoustic signal can be found within narrow frequency bands at about $330 \mathrm{~Hz}$ and $1300 \mathrm{~Hz}$.

Figure $1 \mathrm{~b}$ shows the position of the applied accelerometers and the power distributions $F_{i i}$ of their signals, scaled to the maximum of the strongest signal. Comparison of figures $1 \mathrm{a}$ and $1 \mathrm{~b}$ reveals that the frequency bands around $330 \mathrm{~Hz}$ and $1300 \mathrm{~Hz}$ dominating the acoustic spectrum also appear prominently in the spectra of the lateral mechanical vibrations. Compared to the lateral motion of the outer sensor acc3, the circumferential vibrations of acc 4 and acc 5 are of small power. The fact that the circumferential as well as the lateral motions gain much energy in the band at $330 \mathrm{~Hz}$ suggests a coupling of both motions.

Further analysis clarifies the motion of the caliper: figure $2 b$ and $2 c$ show the positions of the caliper points $1 \ldots 3$ versus time, calculated by filtering and double time-integration of the acceleration data of acc $1 \ldots$ acc 3 . While point 3 undergoes large motions, point 1 almost is in rest. Obviously the caliper tilts about an instantaneous center near point 1 . At the time denoted by the vertical arrow, the brake is relased and oscillations decay due to damping.

* Corresponding author: e-mail: hetzler@itm.uni-karlsruhe.de, Phone: +49721 608 6069, Fax: +49721 6086070 

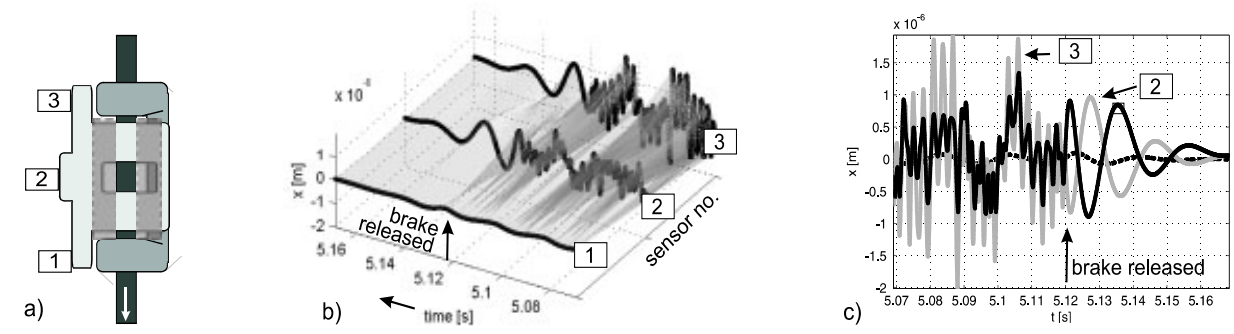

Fig. 2

\section{Minimal lumped parameter model}

A closer look at the functionality of the brake may explain the observed behavior: if the brake is active, the friction forces push the pads against their circumferential supports. Due to contact friction, the pads cannot move sidewards and therefore only can rotate about these support points, causing a deformation and therewith restitutive moment of the disc.

A simple lumped-parameter model covering the observed rotational motion of the caliper and the motion of the pads is depicted in figure 3a. It comprises two masses $m$ representing the pads, which are guided between an outer housing (black,
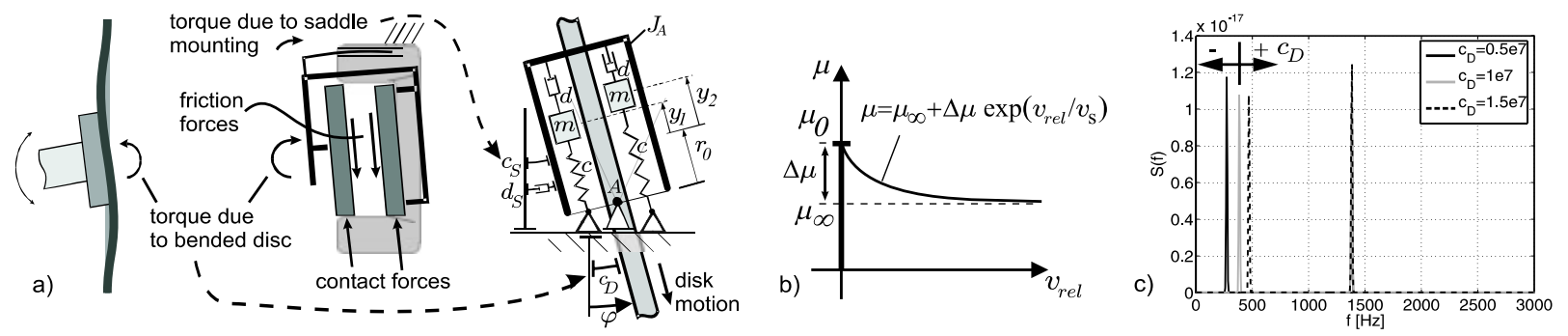

Fig. 3 a) Mechanical model. b) Friction coefficient modelling. c) Simulation result.

thick line) representing the caliper and moving bar (grey) representing the rotating disc in between them. The housing (mass moment of inertia w.r.t. $A$ ) and the disc can tilt about the common center of rotation $A$. The pads are supported by springs (spring constant $c$ ) and dampers (damping coefficient $d$ ). The sliding friction in the contact between each pad and the disc is described as Stribeck friction (cf. 3b); stiction does not occur. The elastic behavior of the disc is taken into account by modelling the normal force in the contact as $N_{1 / 2}=N_{0} \pm c_{D} \varphi$ with a constant brake force $N_{0}$ and a part $\pm c_{D} \varphi$ due to the stiffness of the disc. With $\omega_{S}=\sqrt{\frac{c_{S}}{J_{A}}}, \omega_{D}=\sqrt{\frac{c_{D}}{J_{A}}}, \omega_{0}=\sqrt{\frac{c}{m}}, D_{S}=\frac{d_{S}}{2 m \omega_{S}}, D=\frac{d}{2 m \omega_{0}}$, the equation of motion is

$$
\ddot{\vec{z}}+\left[\begin{array}{ccc}
2 D_{S} \omega_{S} & & \\
& 2 D \omega_{0} & \\
& & 2 D \omega_{0}
\end{array}\right] \dot{\vec{z}}+\left[\begin{array}{ccc}
\omega_{S}^{2}+2 r_{0}^{2} \omega_{D}^{2} & -\frac{N_{0}}{J_{A}} & \frac{N_{0}}{J_{A}} \\
& \omega_{0}^{2} & \omega_{0}^{2}
\end{array}\right] \vec{z}+\vec{f}(\vec{z})=\overrightarrow{0} .
$$

The term $f(z)$ denotes nonlinear terms depending on the state vector $\vec{z}=\left[\varphi, y_{1}, y_{2}\right]^{T}$ and contains e.g. the Stribeck friction and additional coupling terms. Equation (1) represents an autonomous dynamical system, which is able to exhibit self-excited oscillations due to the nonlinear terms in $f(\vec{z})$. The DOF are coupled via the normal force influencing the friction term. Figure 3c exemplarily shows the power spectral density $S_{\varphi \varphi}(f)$ for three different values of disc stiffness $c_{D}$, which directly influences the position of the lower spectral peak. Vice versa, the position of the upper spectral band depends on the stiffness $c$ of the pads. The parameters for the simulation had been chosen according to literature (see e.g. [2], [3]), the disc stiffness was estimated using an FEM calculation: $J_{A}=0.125 \mathrm{kgm}^{2}, m=0.2 \mathrm{~kg}, c=1 \cdot 10^{7} \mathrm{Nm}^{-1}, c_{D}=1.5 \cdot 10^{7} \mathrm{~N}, D=0.001, D=$ $0.01, N_{0}=2000 \mathrm{~N}, r_{0}=0.1 \mathrm{~m}, U=2.2 \mathrm{~min}^{-1}, \mu_{0}=0.4, \mu_{\infty}=0.25, v_{s}=70 \mathrm{~ms}^{-1}$.

Numerical analyses show that the behavior of this model strongly depends on the initial conditions. For example, an initially tilted caliper will trigger coupled oscillations in the lower frequency band, while initial displacements of the pads mainly cause high frequency oscillations. Mixed initial conditions produce both spectral contents. Further work will concentrate on stability, resonance phenomena including energy exchange between the DOF and the influence of nonlinear elastic and damping terms.

\section{References}

[1] Wallaschek, J., et. al.: A Survey Of The Present State Of The Friction Modelling In The Analytical And Numerical Investigation Of Brake Noise Generation; ASME (1999).

[2] Ch. Schmalfuß: Theoretische und experimentelle Untersuchung von Scheibenbremsen, VDI Fortschrittbericht, Reihe 12, 2002

[3] U. von Wagner, P. Hagedorn, et.al.: Brake Squeal: Modelling and Experiments, VDI Bericht, Nr. 1749, S. 173-186, 2003 